\title{
A Survey of Expert Opinion Regarding Rotator Cuff Repair.
}

\author{
Daniel C Acevedo \\ Rothman Institute, Thomas Jefferson University \\ E Scott Paxton \\ Rothman Institute, Thomas Jefferson University \\ Gerald R Williams \\ Rothman Institute, Thomas Jefferson University \\ Joseph A Abboud \\ Rothman Institute, Thomas Jefferson University
}

Follow this and additional works at: https://jdc.jefferson.edu/rothman_institute

Part of the Orthopedics Commons

Let us know how access to this document benefits you

\section{Recommended Citation}

Acevedo, Daniel C; Paxton, E Scott; Williams, Gerald R; and Abboud, Joseph A, "A Survey of Expert Opinion Regarding Rotator Cuff Repair." (2014). Rothman Institute Faculty Papers. Paper

54.

https://jdc.jefferson.edu/rothman_institute/54

This Article is brought to you for free and open access by the Jefferson Digital Commons. The Jefferson Digital Commons is a service of Thomas Jefferson University's Center for Teaching and Learning (CTL). The Commons is a showcase for Jefferson books and journals, peer-reviewed scholarly publications, unique historical collections from the University archives, and teaching tools. The Jefferson Digital Commons allows researchers and interested readers anywhere in the world to learn about and keep up to date with Jefferson scholarship. This article has been accepted for inclusion in Rothman Institute Faculty Papers by an authorized administrator of the Jefferson Digital Commons. For more information, please contact: JeffersonDigitalCommons@jefferson.edu. 


\title{
THE \\ ORTHOPAEDIC FORUM
}

\section{A Survey of Expert Opinion Regarding Rotator Cuff Repair}

\author{
Daniel C. Acevedo, MD, E. Scott Paxton, MD, Gerald R. Williams, MD, and Joseph A. Abboud, MD
}

\begin{abstract}
Many patients with rotator cuff tears have questions for their surgeons regarding the surgical procedure, perioperative management, restrictions, therapy, and ability to work after a rotator cuff repair. The purpose of our study was to determine common clinical practices among experts regarding rotator cuff repair and to assist them in counseling patients. We surveyed 372 members of the American Shoulder and Elbow Surgeons (ASES) and the Association of Clinical Elbow and Shoulder Surgeons (ACESS); 111 members (29.8\%) completed all or part of the survey, and $92.8 \%$ of the respondents answered every question. A consensus response (>50\% agreement) was achieved on $49 \%$ ( 24 of 49) of the questions. Variability in responses likely reflects the fact that clinical practices have evolved over time based on clinical experience.
\end{abstract}

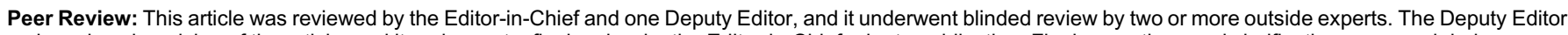

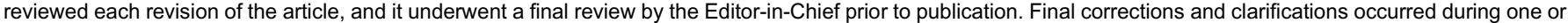
more exchanges between the author(s) and copyeditors.

Rotator cuff tears are a common shoulder problem in up to $54 \%$ of people older than sixty years of age; these tears can be treated successfully both operatively and nonoperatively with good clinical results ${ }^{1,2}$. Recently, the American Academy of Orthopaedic Surgeons published clinical practice guidelines for optimizing the treatment of rotator cuff problems ${ }^{3}$. These guidelines were produced by a volunteer group of shoulder surgeons who performed a systematic review of the current literature regarding the management of rotator cuff pathology. Many of the recommendations were inconclusive because of the lack of high-level evidence.

When strong scientific evidence on a particular topic is not available, providers are forced to make treatment decisions based largely on clinical experience and expert opinion. Many patients have basic questions after a rotator cuff repair pertaining to activities of daily living such as showering and driving, as well as returning to work and recreational activities. High levels of evidence do not support definitive recommendations regarding these perioperative issues. Thus, shoulder surgeons have developed a personal approach to the management of patients with rotator cuff problems. Surgeons who are earlier in their career may not have the clinical experience needed to answer patients' questions and address common clinical concerns based on their personal experience.

Previous studies have utilized web-based surveys to evaluate surgeon practice patterns regarding various shoulder disorders $^{4-6}$. The purpose of our study was to assess the current approach of orthopaedic surgeons specializing in shoulder

Disclosure: None of the authors received payments or services, either directly or indirectly (i.e., via his or her institution), from a third party in support of any aspect of this work. One or more of the authors, or his or her institution, has had a financial relationship, in the thirty-six months prior to submission of this work, with an entity in the biomedical arena that could be perceived to influence or have the potential to influence what is written in this work. No author has had any other relationships, or has engaged in any other activities, that could be perceived to influence or have the potential to influence what is written in this work. The complete Disclosures of Potential Conflicts of Interest submitted by authors are always provided with the online version of the article. 
surgery to important aspects of perioperative clinical management of rotator cuff tears. Our hypothesis was that there would be a majority consensus (of over $50 \%$ agreement) among shoulder surgeons to greater than $50 \%$ of the questions regarding the perioperative counseling and management of patients with rotator cuff disease.

\section{Materials and Methods \\ Survey Population}

An online survey (SurveyMonkey, Portland, Oregon) was created to assess the typical approach that an expert shoulder surgeon uses to address important clinical questions relating to rotator cuff repair and surgeon-driven concerns regarding perioperative management. A link to the survey was sent by e-mail to the 359 members and thirteen affiliate members of the American Shoulder and Elbow Surgeons (ASES) and the Association of Clinical Elbow and Shoulder Surgeons (ACESS) who had e-mail addresses on file as of October 2012.

\section{Survey Detail}

The survey included fifty-two questions. The participants were queried in regard to the type of practice they worked in, the percentage of their practice that was dedicated to shoulder surgery, and their preferred method of rotator cuff repair. The remaining forty-nine questions were formulated by the two senior authors (G.R.W. and J.A.A.) based upon their collective experience of important clinical issues regarding the operative treatment of rotator cuff tears. The content of the forty-nine questions included recommendations on when to resume work, allowable activities of daily living after a rotator cuff repair, postoperative sling use, length and type of postoperative medication use, and lifting guidelines, as well as the discussion of expected postoperative outcomes with patients. The answer choices reflected the manner in which survey participants would answer for patients in their practice and how they would manage their patients postoperatively. The survey included questions regarding postoperative rehabilitation, patient counseling concerning activities of daily living, and perioperative surgical and clinical management (see Appendix 1).

\section{Statistical Analysis}

The survey responses were collected and tabulated anonymously with use of available software provided by the online survey system. Questions were evaluated to determine if there was greater than $50 \%$ respondent agreement, and, if so, this was considered a "consensus" response. Questions that did not fulfill this criterion were evaluated for the most common response, and this information also was reported. We used a multivariate model to determine whether there was any correlation between the questions that achieved a consensus response with the type of practice, proportion of shoulder surgery performed, and the type of rotator cuff surgery (open or arthroscopic). Significance was determined to be a $p$ value of $<0.05$, and the $p$ value thresholds for significance were adjusted for the testing of multiple end points.

\section{Funding}

No funding was necessary for this study.

\section{Results}

\section{Respondents' Characteristics}

Of the 372 members of the ASES and ACESS groups who were contacted by e-mail, $111(29.8 \%)$ completed all or part of the survey. A total of $92.8 \%$ of 111 respondents answered every question.

Of the survey participants, $37.6 \%$ worked in an academic practice, $30.3 \%$ worked in a private practice, and $32.1 \%$ worked in a combination academic-private practice. Of the participating surgeons, $89 \%$ dedicated between $50 \%$ and $100 \%$ of their practice to the treatment of shoulder disorders.

\section{Surgical Management}

For rotator cuff repair, $77.8 \%$ of surgeons used an arthroscopic repair, $15.7 \%$ used an open repair, and $6.5 \%$ used a mini-open repair as their preferred technique. Most repairs were performed in a hospital setting ( $51 \%$ of respondents) or in a surgery center ( $43.1 \%$ of respondents). The remaining $5.9 \%$ performed a comparable amount of rotator cuff surgery in both settings. Additionally, $62.1 \%$ responded that they "almost never" had to admit a patient to the hospital on the evening of surgery for pain control or patient request.

\section{Patient Counseling}

The most common responses regarding healing rates quoted to patients included an $80 \%$ to $90 \%$ healing rate for small tears $(<2 \mathrm{~cm})$ by $50 \%$ of surgeons, a $70 \%$ to $80 \%$ healing rate for large tears $(2$ to $4 \mathrm{~cm}$ ) by $43.1 \%$ of surgeons, and a $50 \%$ to $60 \%$ healing rate for massive tears $(>5 \mathrm{~cm})$ by $49 \%$ of surgeons. A total of $43.1 \%$ of surgeons told their patients that the complication rate after rotator cuff surgery is less than $5 \%$. A total of $69.9 \%$ of survey participants told their patients that their shoulder would be "as good as it gets" one year after surgery. A total of $91.3 \%$ of the survey participants responded that they would perform a rotator cuff repair on a smoker.

\section{Postoperative Care}

An abduction sling with a pillow (size not specified) was recommended by $54.5 \%$ of the respondents for postoperative immobilization; five to six weeks was the most commonly reported time period of recommended sling use for all tear sizes. Sling wear for five to six weeks was recommended by $42.7 \%$ for a small tear, $65 \%$ for a large tear, and $58.3 \%$ after repair of massive tears. Sixty-six percent of respondents do not allow their patients to remove the sling while sitting or sleeping in the immediate postoperative period.

Questions regarding postoperative medication use were directed to the respondents regarding chronic anticoagulants, postoperative deep venous thrombosis (DVT) prophylaxis, narcotic use, and use of nonsteroidal anti-inflammatory drugs (NSAIDs). Patients who were on chronic anticoagulants (e.g., Coumadin [warfarin sodium], Plavix [clopidogrel]) are allowed to restart their medication on the first postoperative day by $48 \%$ of the survey respondents. The majority (86.4\%) of shoulder surgeons who participated in the survey do not use DVT prophylaxis routinely after a rotator cuff repair; $51 \%$ allow their patients to take NSAIDs within the first four weeks after surgery, while $49 \%$ do not. In response to the length of time that a postoperative patient would possibly need to take pain medications, $28.2 \%$ of surgeons quoted three to four weeks. Responses varied greatly ranging from less than one week to more than six weeks, with no clear consensus. Percocet (oxycodone and acetaminophen) was the most common narcotic prescribed for postoperative pain control $(54.5 \%)$. We did not question surgeons regarding the use of adjunctive pain control methods. 
The Journal of Bone \& Joint Surgery $\cdot$ Jbjs.org Volume 96-A · Number $14 \cdot$ July 16, 2014

\section{TABLE I Important Clinical Questions with Majority Agreement (>50\%)}

In general, will you operate on a smoker?

Yes

Do you use DVT prophylaxis (Lovenox, ASA*, Coumadin) routinely after rotator cuff surgery for a majority of your patients? No

Do you routinely use an imaging modality to assess for healing in your postoperative rotator cuffs? And if so, what is your preferred imaging modality?

No

Do you give the patient a VIDEO of their arthroscopic cuff repair?

No

Do you tell your patients to sleep in an inclined position after surgery for their comfort?

Yes

Do you have a lifting guideline you recommend patients to follow at 6 months?

Let pain be your guide

Do you have a lifting guideline you recommend patients to follow at 1 year?

Let pain be your guide

After a rotator cuff repair, when do you tell patients that they should expect to be "as good as it gets"'?

1 year

Do you give the patient a copy of their surgical arthroscopy pictures?

Yes

When do you tell a Workers' Compensation patient that they will be able to return to work (assuming a laborer) after repair of a LARGE tear $(2-4 \mathrm{~cm})$ ?

6 months

In the setting of a full-thickness rotator cuff tear with a significantly degenerated biceps, what is your preferred method of treatment (sex and age not being a factor)?

Tenodesis

Do you allow your patients to take off their slings while sitting down or while sleeping in the immediate postoperative period? No

On average, how long do you have your patients wear a sling after rotator cuff repair for a LARGE tear $(2-4 \mathrm{~cm})$ ?

5-6 weeks

How often do you admit patients to the hospital the night of surgery (for pain control, patient requests, etc.)?

Almost never

On average how long do you have your patients wear a sling after rotator cuff repair for a MASSIVE tear $(>5 \mathrm{~cm})$ ?

5-6 weeks

When do you tell Workers' Compensation patients that they will be able to return to work (assuming a laborer) after repair of a SMALL tear $(<2 \mathrm{~cm})$ ?

6 months

In general, when do you allow patients to drive a car after a rotator cuff repair?

When the patient is out of the sling full time

What type of sling do you place your patient in after a standard rotator cuff repair (supraspinatus)?

Sling with a pillow (i.e., UltraSling)

What pain medications do you prescribe for your patients for postoperative pain control? (May choose more than one)

Percocet

In general, how do you treat full-thickness subscapularis tendon tears?

Arthroscopic

In general, do you allow your patients to take NSAIDs after surgery (within the first 4 weeks)?

Yes

In what setting do you perform the majority of your rotator cuff repairs?

Hospital

51

In general, how long do you follow your postoperative cuff repairs?

6 months to $1 \mathrm{yr}$

What percent healing rates do you quote your patients if any for SMALL tears $(<2 \mathrm{~cm})$ ? $80-90 \%$ 
On average how long do you have your patients wear a sling after rotator cuff repair for a SMALL tear $(<2 \mathrm{~cm})$ ?

5-6 weeks

How many days after surgery do you allow your patients to shower?

On average, how long after surgery do you allow patients to go into water (i.e., getting in a pool/Jacuzzi)? Within:

Two weeks

On average, when do you tell patients that they will start formal physical therapy after a rotator cuff repair for SMALL tears ( $<2 \mathrm{~cm})$ ?

1-2 weeks

On average, when do you tell patients that they will start formal physical therapy after a rotator cuff repair for LARGE tears (2-4 cm)? 5-6 weeks

On average, when do you tell patients that they will start formal physical therapy after a rotator cuff repair for MASSIVE tears (>5 $\mathrm{cm}$ )? $>6$ weeks

Patients frequently sleep upright after rotator cuff surgery in a chair/couch. When do you tell patients that they will be able to sleep in bed (lying down) after rotator cuff surgery?

1-2 weeks

When do you allow your patients to go on flights or long car trips after surgery?

Whenever the patient is comfortable immediately after surgery

In general, when do you allow your patients to return to work at a sedentary job after repair of a small tear $(<2 \mathrm{~cm})$ ?

1-2 weeks

In general, when do you allow your patients to return to work at a sedentary job after repair of a LARGE (2-4 cm) tear? 1-2 weeks

In general, when do you allow your patients to return to work at a sedentary job after repair of a MASSIVE tear (>5 $\mathrm{cm})$ ? 1-2 weeks

In general, when do you allow your patients to return to work at a LABOR job (lifting, pushing, pulling) after repair of a small tear (<2 cm)? 4 months

In general, when do you allow your patients to return to work at a LABOR job (lifting, pushing, pulling) after repair of a LARGE tear (2-4 cm)? 4 months

In general, when do you allow your patients to return to work at a LABOR job (lifting, pushing, pulling) after repair of a MASSIVE tear (>5 cm)? 6 months

In general, how often do you follow your rotator cuffs postoperatively? Until the patient is fully rehabilitated, and then an as needed basis

What is your preferred type of suture anchor?

PEEK

For what duration of time do you tell patients that they will need pain killers after surgery?

3-4 weeks

When do you tell a Workers' Compensation patient that they will be able to return to work (assuming a laborer) after repair of a MASSIVE tear $(>5 \mathrm{~cm})$ ?

6 months

What is your preferred method of tenodesis (generally speaking)?

Arthroscopic tenodesis

Many patients are on blood thinners (Coumadin, Plavix) for various medical reasons. When do you allow patients to restart their blood thinners after surgery?

The first postoperative day

What percent healing rates do you quote your patients, if any, for LARGE tears $(2-4 \mathrm{~cm})$ ? $70-80 \%$

What percent healing rates do you quote your patients, if any, for MASSIVE tears $(>5 \mathrm{~cm})$ ?

$50-60 \%$

What is the rate of complications that you quote for your patients undergoing rotator cuff repair? Less than $5 \%$

Do you have a lifting guideline you recommend patients follow at 1 month? 1 pound

Do you have a lifting guideline you recommend patients follow at 3 months?

10 pounds 


\section{Activities of Daily Living}

A large number of surgeons (55.3\%) do not allow their patients to drive a car until the arm is out of the sling full time. A smaller number $(26.2 \%)$ did allow patients to drive once they were off narcotics but still wearing a sling. There was variability in how many days until showering was allowed postoperatively, with $28.2 \%$ allowing showers at three days. Immersion in a pool or Jacuzzi was allowed at two weeks after surgery by $32 \%$ of respondents, and at three weeks by $27.2 \%$ of respondents, without a consensus. Most of the respondents (73.8\%) instructed their patients to sleep in an inclined position after rotator cuff surgery for comfort. There was variability as to when surgeons advised their patients that they would be able to sleep in a bed comfortably, with $25.2 \%$ stating between one and two weeks, and some quoting that this would be possible within the first week $(23.3 \%)$, with no consensus. According to $49.5 \%$ of the respondents, traveling on planes or on long car trips is allowed immediately after surgery as long as the patient is comfortable.

\section{Return to Work}

Surgeons were asked when they allowed their patients to return to work at a sedentary job and to a manual labor job after repair of small, large, and massive rotator cuff tears. The same question was posed in the setting of Workers' Compensation claims. The most common response in regard to return to a sedentary job was one to two weeks for small tears $(41.2 \%)$, large tears $(38.2 \%)$, and massive tears $(34.3 \%)$.

For a manual labor job, two responses were most common: $34.7 \%$ allow patients to return to work at three months, and $35.6 \%$ allow return to work at four months after repair of small tears. Additionally, $17.8 \%$ of respondents allowed return at six months for small tears. After repair of a large tear or a massive tear, respondents most commonly allow their patients to return to manual labor at four months $(29.4 \%)$ or six months (34\%), respectively.

In regard to patients with Workers' Compensations claims, a large percentage of surgeons do not allow a return to manual labor until six months postoperatively for small tears (56.6\%), although $35.4 \%$ allow an earlier return at three months. There was a consensus with surgeons who allow return to work after six months for large tears (68\%). After repair of a massive tear, $40.2 \%$ allow return to work after at least six months, but $38.2 \%$ responded "maybe never."

\section{Postoperative Rehabilitation}

The survey queried the surgeons on when their patients most commonly start a formal postoperative physical therapy program. For small tears, $26.5 \%$ started therapy within two weeks. Responses varied greatly for large and massive tears. The most common response was five to six weeks for large tears $(26.2 \%)$ and more than six weeks for massive tears $(25.5 \%)$, with no consensus.

In regard to lifting restrictions after repair of any tear size, $37.3 \%$ of surgeons recommended a lifting limit of 1 pound $(0.45 \mathrm{~kg})$ at one month, and $29.4 \%$ allowed 10 pounds ( $4.54 \mathrm{~kg})$ at three months. At six months and one year after surgery, a majority of respondents advised their patients to let pain be their guide as a limit to lifting ( $62.7 \%$ and $72.8 \%$, respectively).

\section{Clinical Follow-up}

With regard to intervals of patient follow-up, there was a split between surgeons who follow their patients every month $(40.8 \%)$ and those who follow their patients "until they are fully rehabilitated and then on an as-needed basis" (41.7\%). In terms of duration of follow-up, $50.5 \%$ of the respondents follow patients between six months and a year postoperatively.

Participants were asked if they routinely use an imaging modality (ultrasound, magnetic resonance imaging, or computed tomography) to assess healing in postoperative patients; $87.4 \%$ responded that they do not. Although $68.6 \%$ of the respondents give their patients a copy of the arthroscopy pictures, $76.7 \%$ do not give the patient a video record of the surgery.

\section{Miscellaneous}

Full-thickness subscapularis tears were treated arthroscopically by $54.4 \%$ of the survey respondents. In the setting of a fullthickness rotator cuff tear with a degenerated biceps, with age and sex not being a factor, $66 \%$ preferred a biceps tenodesis; $38.6 \%$ performed the tenodesis arthroscopically.

\section{Analysis}

Our multivariate statistical model showed that there was a correlation between a preference for open cuff repair and the decision or need to admit a patient overnight after surgery $(\mathrm{p}=$ 0.0039 ). There was also a correlation with open surgery and the method of treatment for full-thickness subscapularis tears $(\mathrm{p}<$ 0.00001). Thus, respondents who preferred open cuff repair were more likely to admit a patient (log odds ratio, 1.5855; standard deviation error, 0.5162) and less likely to repair a subscapularis tear arthroscopically (log odds ratio, -4.3181 ; standard deviation error, 1.1739). The remainder of the twenty-two questions that had a consensus response showed no correlation between the type of practice, proportion of shoulder surgery performed, and the type of rotator cuff surgery (open or arthroscopic).

Our results indicate that our hypothesis was incorrect; there was only a majority consensus for $49 \%$ of the survey questions (twenty-four of forty-nine questions) (Tables I and II). See Appendix 2 for a complete table of responses.

\section{Discussion}

The purpose of our study was to assess the current approach of orthopaedic surgeons specializing in shoulder surgery to important clinical questions after rotator cuff surgery. The responses to twenty-four of forty-nine (49\%) questions achieved a consensus agreement $(>50 \%)$. Eighty-nine percent of the surgeons surveyed dedicate over half of their practices to the treatment of shoulder pathology, supporting the assumption that these physicians are experienced in the treatment of rotator cuff problems 
The Journal of Bone \& Joint Surgery $\cdot$ JbJs.org VOlume 96-A • Number $14 \cdot$ July 16,2014
A Survey of Expert Opinion Regarding Rotator Cuff Repair

One of the most common and important questions posed by patients is, "When can I go back to work?" According to our survey, most shoulder surgeons allow patients to return to work at a sedentary job at one to two weeks after surgery, regardless of the tear size. This time frame increased for manual labor jobs. A patient with a Workers' Compensation claim was typically not allowed to return to full-duty heavy labor until six months, if ever, after repair. This is consistent with what is reported in the literature ${ }^{18}$. Patients with Workers' Compensation claims have a tendency toward inferior results and longer recovery times. It may be that many surgeons wait six months to offer the patient the maximum recovery time in order to achieve the best outcome and ability to return to work. Over one-third of surgeons (38.2\%) stated that it is possible that patients might never return to manual labor after repair of a massive tear. Possible explanations for this include the lower reported healing rates of massive repairs and worry that manual labor will accelerate shoulder degeneration if the repair does not heal ${ }^{19-22}$.

There are obvious limitations to our study. One weakness is the relatively low response rate $(29.8 \%)$. There may be selection bias in those who responded and, therefore, the responses to this survey may not actually reflect the opinion of the majority of the ASES and ACESS members. Another limitation of this study is that it was computer-based and may be biased against those who are less computer-savvy. In addition, the questions in our study were developed based upon the expert opinion of two surgeons and were not developed through a validated process or methodology. Lastly, the information we obtained from the survey is largely based on expert opinion and personal practice patterns.

We concluded that the responses to important clinical questions by expert shoulder surgeons are varied, with less than half of our questions achieving a majority response $(>50 \%)$. Given the variability of responses in this study, the management of perioperative clinical questions is likely based on personal experience. The results of this survey may offer some guidance to managing these clinical questions as well as guide future research into topics with widely variable responses.

\section{Appendices}

eA The survey instrument and the complete survey responses are shown in Appendix 1 and Appendix 2, respectively, and are available with the online version of this article as a data supplement at jbjs.org.

responses concerning the onset of therapy postoperatively were varied, regardless of the size of the rotator cuff tear. Recent studies have found limited benefit to early range of motion after rotator cuff repair, with some concern of possible increased likelihood of retear with earlier motion, especially in patients with larger tears ${ }^{15,16}$. Some surgeons believe that a delay in rehabilitation is beneficial for healing and does not cause long-term stiffness ${ }^{17}$; this opinion is reflected in our study. It is also conceivable that timing to passive range-of-motion therapy has no impact on healing rate or outcome after a repair, so the decision to move early is inconsequential, possibly explaining the variability in responses.
Daniel C. Acevedo, MD

E. Scott Paxton, MD

Gerald R. Williams, MD

Joseph A. Abboud, MD

The Rothman Institute at Thomas Jefferson University, 925 Chestnut Street,

Philadelphia, PA 19107.

E-mail address for D.C. Acevedo: Acevedomd@gmail.com 


\section{References}

1. Bartolozzi A, Andreychik D, Ahmad S. Determinants of outcome in the treatment of rotator cuff disease. Clin Orthop Relat Res. 1994 Nov;(308):90-7.

2. Aleem AW, Brophy RH. Outcomes of rotator cuff surgery: what does the evidence tell us? Clin Sports Med. 2012 Oct;31(4):665-74.

3. Pedowitz RA, Yamaguchi K, Ahmad CS, Burks RT, Flatow EL, Green A, lannotti JP, Miller BS, Tashijian RZ, Watters WC 3rd, Weber K, Turkelson CM, Wies JL, Anderson S, St Andre J, Boyer K, Raymond L, Sluka P, McGowan R; American Academy of Orthopaedic Surgeons. Optimizing the management of rotator cuff problems. J Am Acad Orthop Surg. 2011 Jun;19(6):368-79.

4. Redfern J, Burks R. 2009 survey results: surgeon practice patterns regarding arthroscopic surgery. Arthroscopy. 2009 Dec;25(12):1447-52.

5. Randelli P, Arrigoni P, Cabitza F, Ragone V, Cabitza P. Current practice in shoulder pathology: results of a web-based survey among a community of 1,084 orthopedic surgeons. Knee Surg Sports Traumatol Arthrosc. 2012 May;20(5):803-15. Epub 2011 Oct 01. 6. Golant A, Christoforou D, Zuckerman JD, Kwon YW. Return to sports after shoulder arthroplasty: a survey of surgeons' preferences. J Shoulder Elbow Surg. 2012 Apr;21(4):554-60. Epub 2011 Mar 09.

7. Edgar R, Nagda S, Huffman R, Namdari S. Pulmonary embolism after shoulder arthroscopy. Orthopedics. 2012 Nov;35(11):e1673-6.

8. Kurmis AP, Kurmis TP, O'Brien JX, Dalén T. The effect of nonsteroidal antiinflammatory drug administration on acute phase fracture-healing: a review. J Bone Joint Surg Am. 2012 May 2;94(9):815-23.

9. Cohen DB, Kawamura S, Ehteshami JR, Rodeo SA. Indomethacin and celecoxib impair rotator cuff tendon-to-bone healing. Am J Sports Med. 2006 Mar;34(3):362-9. Epub 2005 Oct 06.

10. Neyton L, Godenèche A, Nové-Josserand L, Carrillon Y, Cléchet J, Hardy MB. Arthroscopic suture-bridge repair for small to medium size supraspinatus tear: healing rate and retear pattern. Arthroscopy. 2013 Jan;29(1):10-7. Epub 2012 Nov 15. 11. Baumgarten KM, Gerlach D, Galatz LM, Teefey SA, Middleton WD, Ditsios K, Yamaguchi K. Cigarette smoking increases the risk for rotator cuff tears. Clin Orthop Relat Res. 2010 Jun;468(6):1534-41. Epub 2009 Mar 13.

12. Carbone S, Gumina S, Arceri V, Campagna V, Fagnani C, Postacchini F. The impact of preoperative smoking habit on rotator cuff tear: cigarette smoking influ- ences rotator cuff tear sizes. J Shoulder Elbow Surg. 2012 Jan;21(1):56-60. Epub 2011 Apr 27.

13. Galatz LM, Silva MJ, Rothermich SY, Zaegel MA, Havlioglu N, Thomopoulos S. Nicotine delays tendon-to-bone healing in a rat shoulder model. J Bone Joint Surg Am. 2006 Sep;88(9):2027-34.

14. Mallon WJ, Misamore G, Snead DS, Denton P. The impact of preoperative smoking habits on the results of rotator cuff repair. J Shoulder Elbow Surg. 2004 Mar-Apr;13(2):129-32.

15. Cuff DJ, Pupello DR. Prospective randomized study of arthroscopic rotator cuff repair using an early versus delayed postoperative physical therapy protocol. J Shoulder Elbow Surg. 2012 Nov;21(11):1450-5. Epub 2012 May 02.

16. Lee BG, Cho NS, Rhee YG. Effect of two rehabilitation protocols on range of motion and healing rates after arthroscopic rotator cuff repair: aggressive versus limited early passive exercises. Arthroscopy. 2012 Jan;28(1):34-42. Epub 2011 Oct 20.

17. Parsons BO, Gruson KI, Chen DD, Harrison AK, Gladstone J, Flatow EL. Does slower rehabilitation after arthroscopic rotator cuff repair lead to long-term stiffness? J Shoulder Elbow Surg. 2010 Oct;19(7):1034-9. Epub 2010 Jul 24.

18. Bhatia S, Piasecki DP, Nho SJ, Romeo AA, Cole BJ, Nicholson GP, Boniquit N, Verma NN. Early return to work in workers' compensation patients after arthroscopic full-thickness rotator cuff repair. Arthroscopy. 2010 Aug;26(8):1027-34. Epub 2010 Jun 03.

19. Burkhart SS, Danaceau SM, Pearce CE Jr. Arthroscopic rotator cuff repair: Analysis of results by tear size and by repair technique-margin convergence versus direct tendon-to-bone repair. Arthroscopy. 2001 Nov-Dec;17(9):905-12.

20. Gerber C, Fuchs B, Hodler J. The results of repair of massive tears of the rotator cuff. J Bone Joint Surg Am. 2000 Apr;82(4):505-15.

21. Yoo JC, Ahn JH, Koh KH, Lim KS. Rotator cuff integrity after arthroscopic repair for large tears with less-than-optimal footprint coverage. Arthroscopy. 2009 Oct;25(10):1093-100

22. Burkhart SS, Barth JR, Richards DP, Zlatkin MB, Larsen M. Arthroscopic repair of massive rotator cuff tears with stage 3 and 4 fatty degeneration. Arthroscopy. 2007 Apr;23(4):347-54 